\title{
FULLY PARAMETRIZED STATE-SPACE MODELS IN SYSTEM IDENTIFICATION
}

\author{
T. MCKELVEY \\ Department of Electrical Engineering, Linköping University, S-581 83 Linköping, Sweden.
}

\begin{abstract}
In this paper we consider identification of multivariable linear systems using state-space models. A new model structure which is fully parametrized is introduced. All systems of a given order can be described with this model structure and thus relieve us from all the internal structural issues otherwise inherent in the multivariable state-space identification problem. We present an identification algorithm which minimize a regularized prediction error criterion. We show that the proposed model structure retains the statistical properties of the standard identifiable model structures. The proposed identification algorithm is shown to locally converge to the set of true systems. Examples are given illustrating the results as well as showing the practical use of the proposed model structure using real data.
\end{abstract}

Key Words. System identification, multivariable systems, state-space models, parameter estimation

\section{INTRODUCTION}

In this paper we consider identification of multivariable linear time invariant finite dimensional systems. We also assume that we have no structural information and thus have to resort to so called black-box models. These can be either of input-output type or of state-space type. There are several reasons that favor the statespace form: (1) They are their own economic implementation algorithm. (2) They have simple relationships between simulation and prediction applications. (3) Many control design methods and algorithms, e.g. the recent Doyle-Glover algorithm solving the $H_{\infty}$ optimal control problem Doyle et al. (1989), use state-space descriptions of the plants to be controlled. (4) From the point of view of computer implementation it is important to use models which have low sensitivity with respect to round-off errors and finite word length effects. Some realizations of state-space models are well behaved in this respect Mullis and Roberts (1976); Thiele (1984); Thiele (1986).

We shall thus consider model structures in statespace innovations form:

$$
\begin{array}{ll}
x(t+1) & =A(\theta) x(t)+B(\theta) u(t)+K(\theta) e(t) \\
y(t) & =C(\theta) x(t)+e(t)
\end{array}
$$

where the matrices $A, B, C$ and $K$ are functions of a parameter vector $\theta, u(t)$ is the input vector of dimension $m, y(t)$ is the output vector of dimension $p$ and $e(t)$ is the noise vector of dimension $p$. In the process of identification we try to determine these matrices by estimating the vector $\theta$ from input-output data.
The task of identification can thus be divided into two steps:

1. Determine a parametrization, i.e. how shall the matrices $A, B, C$ and $K$ depend on the parameter vector $\theta$.

2. Estimate the parameter vector $\theta$ from data.

To solve the second step we will minimize the norm of the prediction errors: Minimize

$$
V_{N}(\theta)=\frac{1}{N} \sum_{t=1}^{N}|\varepsilon(t, \theta)|^{2}
$$

where

$$
\begin{gathered}
\varepsilon(t, \theta)=y(t)-\hat{y}(t \mid \theta) \\
\hat{x}(t+1 \mid \theta)=(A(\theta)-K(\theta) C(\theta)) \hat{x}(t \mid \theta) \\
\hat{y}(t \mid \theta) \quad=\quad+B(\theta) u(t)+K(\theta) y(t) \\
\end{gathered}
$$

See, e.g. Ljung (1987). The minimization of (2) is carried out numerically by an iterative procedure.

We will mainly concentrate on the parametrization problem of multivariable state-space systems. After some notations in Section 2, we will, in Section 3, introduce a fully parametrized state-space model structure which immediately solves the difficult parametrization problem and which also enable us to obtain models with good numerical properties. In Section 4 we give an identification algorithm which is analyzed in 
Section 5. A stochastic analysis is performed in Section 6 and some examples are presented in Section 7 .

\section{NOTATIONS}

A model structure $\mathcal{M}$ is a mapping from the parameter space to the model (1). Let $\mathcal{M}(\theta)$ denote the model obtained from a model structure and a parameter value $\theta$. The dimension of the parameter vector $\theta$ is denoted by $d_{\mathcal{M}}$. Let

$$
D_{\mathcal{M}}=\left\{\theta \in \mathbb{R}^{d_{\mathcal{M}}} \mid \text { The predictor (4) is stable }\right\}
$$

denote the set of possible values of $\theta$. Let $\mathcal{S}=$ $\mathcal{M}\left(\theta_{0}\right)$ denote the true system. Let

$$
D_{T}=\left\{\theta \in D_{\mathcal{M}} \mid \mathcal{S}=\mathcal{M}(\theta)\right\}
$$

denote the set of parameters that represents the true system. The model set given by a model structure and a parameter space is

$$
\mathcal{M}^{*}=\left\{\mathcal{M}(\theta) \mid \theta \in D_{\mathcal{M}}\right\} .
$$

Let $Z^{N}$ denote the data set consisting of $\{y(t)\}$ and $\{u(t)\}, t=1, \ldots, N$.

\section{MODEL PARAMETRIZATION}

The parametrization of models to be used in system identification is often closely tied to the concept of identifiability, i.e if two models from the same model structure are equal then this implies that the corresponding parameter vectors are equal. See Ljung (1987) for a formal definition.

Traditionally, see Ljung (1987) and Söderström and Stoica (1989), model structures which are identifiable have been favored for the purpose of system identification. The use of an identifiable model structure has some clear advantages; the criterion $V_{N}(\theta)$ has a unique minimum if the data is informative, numerical algorithms perform well etc. However the parametrization of multivariable state-space models is a well-known and notoriously difficult problem in system identification, see e.g. Kailath (1980), Luenberger (1967) or van Overbeek and Ljung (1982). The root of the problem is that there is not one single, smooth identifiable parametrization of all multi-output systems (1). Typically one has to work with a large number of different possible parametrizations depending on the internal structure of the system.

The concept of identifiable model structures is important if we are interested in the values of the parameters themselves, e.g. change detection techniques where a change in the parameters indicates a change in the underlying system. If we are only interested in the input/output relation $G(q, \theta)$ the value of each parameter is then of no interest to us. The parameters can be seen as vehicles to describe the interesting characteristics of the system. It is also known that some systems described in their canonical forms have transfer functions with a very high sensitivity with respect to the parameters and are thus sensitive to finite word length effects occurring in a computer, see Thiele (1984). Based on these arguments we will introduce a new model structure which will circumvent these problems.

\subsection{The Fully Parametrized State Space Model}

If we consider the state space model (1) and choose to fill all the matrices $A, B, C, K$ with parameters, we will clearly over-parametrize the model and thus lose identifiability. To formalize:

Let all the matrices $A(\theta), B(\theta), C(\theta)$ and $K(\theta)$ in the model (1) be filled with parameters from the parameter vector $\theta \in D_{\mathcal{M}}$ and let the corresponding model structure be called fully parametrized.

The number of parameters needed for this model structure (5) is

$$
d_{\mathcal{M}_{F}}=n^{2}+2 n p+n m
$$

This fully parametrized model has an additional $n^{2}$ number of parameters compared to the identifiable models given in Ljung (1987), which each have

$$
d_{\mathcal{M}_{I}}=2 n p+n m
$$

number of parameters.

Using this model structure for the purpose of identification will give two interesting implications. First, this state-space model structure would relieve us from the search through the different identifiable models when dealing with multivariable systems since the proposed model structure trivially includes all possible systems of a given order $n$. Secondly, the quality of the estimated model might increase if we use a flexible model structure which not only can describe the underlying system but also allows a numerically well-conditioned description. This is a major difference compared with the identifiable model structures, which by definition only have one realization for each system. Since we are confined to computers with limited accuracy for all calculations it is important to be able to use models which are numerically well conditioned.

With the proposed model structure the minimum of $V_{N}(\theta)$ will now be a surface in the parameter space and thus not unique. This follows from the fact that there exist infinitely many parameter values $\theta$ which yield the same inputoutput properties. This non-uniqueness usually leads to convergence problems if standard optimization algorithms, e.g Newton-type algorithms, are used in the minimization of (2) since most algorithms use the inverse of the Hessian

$$
\frac{d^{2}}{d \theta^{2}} V_{N}(\theta)
$$


and in this case the Hessian will be singular or indefinite and thus non-invertible. One possibility to overcome this problem is to further constrain the solution. We will here focus on regularization which is a standard technique for ill-conditioned estimation problems, see Draper and Nostrand (1979).

Regularization means that the criterion (2) is augmented with a term:

$$
W_{N}^{\delta}(\theta)=V_{N}(\theta)+\frac{\delta}{2}\left|\theta-\theta^{\#}\right|^{2} ; \delta>0
$$

where $|\cdot|$ denotes the Euclidean norm. The regularization term $\delta / 2\left|\theta-\theta^{\#}\right|^{2}$ will have the effect that the Hessian is changed to

$$
\frac{d^{2}}{d \theta^{2}} W_{N}^{\delta}(\theta)=\frac{d^{2}}{d \theta^{2}} V_{N}(\theta)+\delta I .
$$

Notice that the approximate Hessian used in a Gauss-Newton algorithm now will be positive definite. The resulting estimate

$$
\hat{\theta}_{N}^{\delta}=\arg \min _{\theta \in D_{\mathcal{M}}} W_{N}^{\delta}(\theta)
$$

will be a compromise between minimizing $V_{N}(\theta)$ and being close to the value $\theta^{\#}$. Since our objective is to find the $\theta$ which minimizes $V_{N}(\theta)$ it is clear that the choice of $\theta^{\#}$ will influence the result. In the next two section we will address this question together with a presentation and analysis of the identification algorithm.

\section{THE ALGORITHM}

In this section we will present an algorithm for the purpose of identification using the proposed fully parametrized model structure. The algorithm is designed with the goal of providing accurate models with low sensitivity with respect to finite word length representations.

In Thiele (1986) results are given which show that a balanced realization, see Moore (1981), is a realization with minimum sensitivity with respect to finite word length effects in the parameters and round off errors. These results leads us to find an algorithm which has a balanced realization as the convergence point. This is easily achieved if we solve a sequence of minimizations of (8) and in each step adjust the vector $\theta^{\#}$ to be a balanced realization of the last estimate. This means that we not only obtain a balanced realization in the limit but also use it in each step of the identification. This gives the following algorithm:

\section{Algorithm 4.1}

1. Let $\hat{\theta}_{b}^{0}$ be initialized with an ARX estimate of the system in a balanced realization.

2. Solve the minimization problem

$$
\hat{\theta}^{k}=\arg \min _{\theta \in D_{\mathcal{M}}} V_{N}(\theta)+\frac{\delta}{2}\left|\theta-\hat{\theta}_{b}^{k-1}\right|^{2} .
$$

3. Convert the obtained estimate to represent a balanced realization.

$$
\hat{\theta}_{b}^{k}=b\left(\hat{\theta}^{k}\right)
$$

4. Repeat steps $2-3$ until $\left|\hat{\theta}^{k+1}-\hat{\theta}^{k}\right|$ is sufficiently small.

The ARX estimate in step 1 is obtained as a least-squares solution. If a multivariable system is at hand, the initial order of the ARX model should be chosen as $n p$ and then followed by a model reduction step to get the model order $n$. Step 2 of the algorithm can be easily be solved by a Gauss-Newton method, see Dennis and Schnabel (1983). The balanced realization is obtained via singular value decompositions, see Moore (1981).

\section{CONVERGENCE ANALYSIS}

In this section we will analyze the convergence properties of Algorithm 4.1.

We will restrict the analysis to local behavior and can thus simplify the calculations by considering a linear regression setup: Let the data $Z^{\infty}$ be generated by the system

$$
y(t)=\psi(t)^{T} \theta_{0}+e(t)
$$

where the regressors $\psi(t)$ are made up from data and the noise terms $e(t)$ are assumed to be a sequence of independent stochastic variables with zero mean $E e(t)=0$, covariance $E e(t) e(t)^{T}=\Lambda$ and independent of the regressors. Let the prediction model be

$$
\hat{y}(t \mid \theta)=\psi(t)^{T} \theta
$$

Consider the criterion

$$
\bar{V}(\theta)=\lim _{N \rightarrow \infty} E V_{N}(\theta) .
$$

This gives us

$$
\bar{V}(\theta)=\operatorname{tr} \Lambda+\left(\theta_{0}-\theta\right)^{T} Q\left(\theta_{0}-\theta\right)
$$

with

$$
Q=\lim _{N \rightarrow \infty} E \sum_{k=1}^{N} \psi(t) \psi(t)^{T} .
$$

Now assume that the matrix $Q$ is only positive semidefinite which implies that $\bar{V}(\theta)$ obtains its minimum value $\operatorname{tr} \Lambda$ for all $\theta=\theta_{0}+z, z \in \mathcal{N}(Q)$ the null space of the matrix $Q$.

If we let the data set $Z^{\infty}$ be informative, the set $D_{T}$ for this system is then given by

$$
D_{T}=\left\{\theta \mid \theta=\theta_{0}+z, z \in \mathcal{N}(Q)\right\} .
$$

Consider the sequence of estimates

$$
\hat{\theta}^{k}=\arg \min _{\theta \in D_{\mathcal{M}}} \bar{V}(\theta)+\delta\left|\theta-\hat{\theta}^{k-1}\right|^{2} .
$$


Theorem 5.1 Let the data set $Z^{\infty}$ be generated by (10) and let the predictor be given by (11). Let the sequence of estimates be given by (14) with $\delta>0$, and let $D_{T}$ be given by (13). Then

$$
\lim _{k \rightarrow \infty} \inf _{\bar{\theta} \in D_{T}}\left|\hat{\theta}^{k}-\bar{\theta}\right|=0 .
$$

Proof: See McKelvey (1993).

If we now return to the state-space model structures and consider a Taylor expansion of $\bar{V}(\theta)$ around a $\theta_{0} \in D_{T}$ we get

$$
\begin{gathered}
\bar{V}(\theta) \approx \bar{V}\left(\theta_{0}\right)+\left(\theta-\theta_{0}\right)^{T} \bar{V}^{\prime}\left(\theta_{0}\right)+\frac{1}{2}\left(\theta-\theta_{0}\right)^{T} Q\left(\theta-\theta_{0}\right) \\
Q=\bar{V}^{\prime \prime}\left(\theta_{0}\right)
\end{gathered}
$$

for $\theta$ in the neighborhood of $\theta_{0}$. The second term is zero since $\theta_{0} \in D_{T}$ is a minimum. If all higher order terms are small the approximation is valid locally and we have the same problem as for the linear case given in Theorem 5.1. This implies that the iterative minimization scheme in Algorithm 4.1 will also be locally convergent for the fully parametrized state-space model structures which exhibit the same non-uniqueness properties as the linear regression setup in the theorem.

\section{ANALYSIS OF THE PREDICTION QUALITY}

In system identification literature the concept of parsimony is considered to be very important, see Söderström and Stoica (1989); Ljung (1987). Use the simplest model with as few parameters as possible. This concept can easily be justified by analyzing the statistical properties of the prediction error. The key result is that the variance of each estimated parameter increases if we estimate more parameters with a fixed amount of data and thus degrade the quality of the estimated model. An identifiable model of a given order has, by definition, a minimal number of parameters and thus satisfies this concept. Even though the fully parametrized model has more parameters than the corresponding identifiable model we will in this section show that regularization will restore the statistical properties of an identifiable model.

Let $\mathcal{M}_{F}$ be the fully parametrized model structure and let $\mathcal{M}_{I}$ denote one of the identifiable model structures both of order $n$. It can then be shown that there exists a differentiable function

$$
g(\theta): \tilde{D}_{\mathcal{M}_{F}} \rightarrow D_{\mathcal{M}_{I}}
$$

such that

$$
\mathcal{M}_{F}(\theta)=\mathcal{M}_{I}(g(\theta)), \quad \forall \theta \in \tilde{D}_{\mathcal{M}_{F}}
$$

where $\tilde{D}_{\mathcal{M}_{F}} \subset D_{\mathcal{M}_{F}}$ is the set of parameters which yield all models in the model set $\mathcal{M}_{I}^{*}$
The gradient of the predictor $\hat{y}(t \mid \theta)$ is defined as

$$
\psi(t, \theta)=\frac{d}{d \theta} \hat{y}(t \mid \theta)
$$

a matrix of dimension $d_{\mathcal{M}} \times p$. Independent of the parameterization, the gradient has the following property:

Lemma 6.1 Let the predictor $\hat{y}^{F}\left(t \mid \theta_{F}\right)$ be given by a fully parametrized model structure (5). Then

$$
\operatorname{rank} \frac{1}{N} \sum_{t=1}^{N} \psi^{F}\left(t, \theta_{F}\right) \psi^{F}\left(t, \theta_{F}\right)^{T} \leq d_{\mathcal{M}_{I}}
$$

where $\psi$ is given by (15) and $d_{\mathcal{M}_{I}}$ by (7).

Proof. Pick a predictor $\hat{y}^{I}\left(t, \theta_{I}\right)$ from an identifiable model structure such that

$$
\hat{y}^{I}\left(t, \theta_{I}\right)=\hat{y}^{F}\left(t, \theta_{F}\right), \quad \forall t .
$$

Then we have

$$
\begin{aligned}
\psi^{F}\left(t, \theta_{F}\right) & =\frac{d}{d \theta_{F}} \hat{y}^{F}\left(t \mid \theta_{F}\right)=\frac{d}{d \theta_{F}} \hat{y}^{I}\left(t \mid g\left(\theta_{F}\right)\right) \\
& =\frac{\partial}{\partial \theta_{F}} g\left(\theta_{F}\right) \cdot \psi^{I}\left(t, \theta_{I}\right)
\end{aligned}
$$

which gives us

$$
\begin{gathered}
\frac{1}{N} \sum_{t=1}^{N} \psi^{F}\left(t, \theta_{F}\right) \psi^{F}\left(t, \theta_{F}\right)^{T}=\frac{\partial}{\partial \theta_{F}} g\left(\theta_{F}\right) . \\
\cdot\left[\frac{1}{N} \sum_{t=1}^{N} \psi^{I}\left(t, \theta_{I}\right) \psi^{I}\left(t, \theta_{I}\right)^{T}\right] \cdot\left(\frac{\partial}{\partial \theta_{F}} g\left(\theta_{F}\right)\right)^{T} .
\end{gathered}
$$

The proof is concluded by observing that $\psi^{I}\left(t, \theta_{I}\right) \psi^{I}\left(t, \theta_{I}\right)^{T}$ has dimension $d_{\mathcal{M}_{I}} \times d_{\mathcal{M}_{I}}$ and applying Sylvester's inequality.

In the main result the matrix

$$
\begin{gathered}
Q=\frac{d^{2}}{d \theta^{2}} \bar{V}\left(\theta_{0}\right) \\
=\lim _{N \rightarrow \infty} E \frac{2}{N} \sum_{t=1}^{N} \psi\left(t, \theta_{0}\right) \psi\left(t, \theta_{0}\right)^{T}
\end{gathered}
$$

of dimension $d_{\mathcal{M}_{F}} \times d_{\mathcal{M}_{F}}, \theta_{0} \in D_{T}$ plays an important part. From Lemma 6.1 it directly follows that

$$
\operatorname{rank} Q \leq d_{\mathcal{M}_{I}}
$$

Assumption A1 Consider a data set $Z^{\infty}$ which has been generated by the system (1) with $\mathrm{e}(\mathrm{t})$ a sequence of independent stochastic variables with zero mean and covariance $\Lambda$. Furthermore assume that the input $u(t)$ is chosen so the data set is informative and that the data 
set satisfies the technical condition D1 in Ljung (1987), p. 210.

For the definition of informative we refer to Ljung (1987). If we now consider the regularized version of the criterion (8) and let $\theta^{\#}=\theta_{0} \in D_{T}$ we have:

Theorem 6.1 Let $\hat{\theta}_{N}^{\delta}$ be defined by (8) and (9) with $\theta^{\#}=\theta_{0} \in D_{T}$ and let the data $Z^{\infty}$ satisfy A1. Then

$$
\hat{\theta}_{N}^{\delta} \rightarrow \theta_{0}, \text { w.p. } 1 \text { as } N \rightarrow \infty
$$

Proof. Immediate from Theorem 8.3 in Ljung (1987) and the choice of $\theta^{\#}$.

We will now present a statistical model quality measure for the fully parametrized models obtained via minimization of the regularized prediction error criterion (8) and (9) with $\theta^{\#}=\theta_{0} \in D_{T}$. For a finite data set $Z^{N}$ the obtained estimate $\hat{\theta}_{N}^{\delta}$ will deviate a little from $\theta_{0}$. As a measure of the quality of the estimated model using finite data we consider the scalar

$$
\bar{V}\left(\hat{\theta}_{N}^{\delta}\right)=\lim _{N \rightarrow \infty} E V_{N}\left(\hat{\theta}_{N}^{\delta}\right)
$$

For all estimates we have $\bar{V}\left(\hat{\theta}_{N}^{\delta}\right) \geq \bar{V}\left(\theta_{0}\right)=$ $\operatorname{tr}(\Lambda), \theta_{0} \in D_{T}$ and the increase of $\bar{V}\left(\hat{\theta}_{N}^{\delta}\right)$ due to the parameter deviation should be as low as possible. This assessment criterion thus considers the variance of the one-step prediction errors, when the estimated model is applied to future data. Finally we introduce

$$
\bar{V}_{N}^{\delta}=E \bar{V}\left(\hat{\theta}_{N}^{\delta}\right)
$$

with the expectation taken over the estimated parameters, as a performance measure "on the average" for estimated models.

We now give the main result which is proven in McKelvey (1993).

Theorem 6.2 Consider the fully parametrized model structure (5) and let the data set $Z^{\infty}$ satisfy $A 1$ and be generated by a true system with $\Lambda=\lambda_{0} I$. Furthermore let $\hat{\theta}_{N}^{\delta}$ be given by (8-9) where $\theta^{\#}=\theta_{0} \in D_{T}$. Then

$$
\lim _{\delta \rightarrow 0} \bar{V}_{N}^{\delta}=\lambda_{0}\left(p+\frac{\operatorname{rank} Q}{N}\right) .
$$

where $\bar{V}_{N}^{\delta}$ is given by (19) and (20) and $p$ is the number of outputs.

The expression (21) is similar to the known expression

$$
\bar{V}_{N}^{\delta}=\lambda_{0}\left(p+\frac{d_{\mathcal{M}}}{N}\right)
$$

which is valid for identifiable model structures and clearly shows the relation to model accuracy versus number of estimated parameters, see Ljung (1987); Söderström and Stoica (1989). However Theorem 6.2 shows us that the fully parametrized model retains the same properties since the rank of $Q$, by Lemma 6.1 , can at most be $d_{\mathcal{M}_{I}}$ which is the number of parameters in a corresponding identifiable model. Hence the fully parametrized prediction model in fact obtains the same statistical properties, i.e. the same prediction error variance, as an identifiable model.

\section{EXAMPLES}

Example 7.1 In this example we will illustrate how different types of parameterizations influence the quality of the identified model if the parameters have finite precision.

Consider the simple third order discrete time system

$$
y(t)=\frac{1}{(q-0.98)^{3}} u(t)+e(t)
$$

where $q$ is the forward time shift operator and $e(t)$ is a sequence of zero mean normal distributed random variables with covariance $10^{-4}$. With $u(t)$ taken as a random signal with unit variance we used the system (23) to generate 100 data sets each with 500 samples of $u(t)$ and $y(t)$. Using these 100 data sets we estimated two different kinds of outputerror models; the fully parametrized state-space model and a state-space model with a minimal parametrization in observability canonical form. For each data record an initial ARX model was estimated. This estimate was then converted to the two different model structures. The fully parametrized model was minimized according to Algorithm 4.1 whereas the canonical model was estimated by minimizing (2). During the estimation we restricted the parameters in the vector $\theta$ to only 5 significant digits. To evaluate the quality of each model we calculated the fit between a simulation of the estimated model $\hat{y}(t)$ and a noise free simulation of the true system (23) using the same input realization.

$$
\mathrm{FIT}=\sqrt{\frac{1}{N} \sum_{t=1}^{N}(y(t)-\hat{y}(t))^{2}}
$$

The mean value calculated over the 100 estimations were 0.91 for the fully parametrized model and 890 for the canonical model. The extreme difference is quite striking since we only used a third order model and we allowed 5 significant digits in all parameters. However from the point of view of information theory the result is quite natural since we used only 6 parameters in the canonical model compared to 21 in the fully parametrized model. The discrete set of possible models is thus much larger for the fully parametrized model set than for the canonical model set.

Example 7.2 In Van Overschee and De Moor (1992) an identification of a glass oven using 
Table 1. Performance $\mathrm{P}$ of the estimated models

\begin{tabular}{ccc}
\hline Model & Sim. & Pred. \\
\hline Subspace & 0.536 & 0.108 \\
Full param. with $K$ & 0.547 & 0.135 \\
Full param. $K=0$ & 0.511 & - \\
\hline
\end{tabular}

real data is given as an example to illustrate the subspace identification algorithm presented in the paper. The system consists of 3 inputs and 6 outputs and is modeled as a system of order $n=5$. Using the same data and our fully parametrized model structure we identified two different models, one output error model, i.e. $K=0$ and one model where $K$ also was estimated. In Table 1 a prediction error measure $P$ of the two estimated models are compared with the model obtained by Van Overschee and De Moor (1992), where

$$
P=\frac{1}{6} \sum_{k=1}^{6} \sqrt{\frac{1}{N} \sum_{t=1}^{N} \frac{\left(y_{k}(t)-\hat{y}_{k}(t)\right)^{2}}{y_{k}(t)^{2}}}
$$

is evaluated on independent data. The fully parametrized OE-model has the best performance of the models in simulations. The other model is a little worse compared to the subspace model. This example shows that identification using real data works quite well with the proposed model structure compared with the subspace identification method.

\section{CONCLUSIONS}

In this paper we have introduced a state space model structure which is fully parametrized and thus each parameter is not identifiable. The use of this model structure for identification of multivariable systems will relieve us from the search through all possible identifiable parametrizations.

In order to minimize the prediction error for the fully parametrized model with an efficient numerical method, regularization is introduced. It is shown that the use of regularization will automatically give the same statistical properties as an identifiable model structure even though the fully parametrized model structure contains more parameters to estimate.

An identification algorithm is presented which yields, in the limit, a balanced realization of the true system which has low sensitivity with regard to finite word length effects. The proposed algorithm thus produces a numerically sound model of the underlying system.

\section{REFERENCES}

Dennis, J. E. and Schnabel, R. B. (1983). Numerical Methods for Unconstrained Optimization and Nonlinear Equations. Prentice-Hall, Englewood Cliffs, New Jersey.

Doyle, J. C., Glover, K., Khargonekar, P. P., and Francis, B. A. (1989). State-space solutions to standard $H_{2}$ and $H_{\infty}$ control problems. IEEE Trans. on Automatic Control, 34(8):832-847.

Draper, N. R. and Nostrand, R. C. V. (1979). Ridge regression and James-stein estimation: Review and comments. Technometrics, 21(4):451-466.

Kailath, T. (1980). Linear Systems. PrenticeHall, Englewood Cliffs, New Jersey.

Ljung, L. (1987). System Identification: Theory for the User. Prentice-Hall, Englewood Cliffs, New Jersey.

Luenberger, D. G. (1967). Canonical forms for linear multivariable systems. IEEE Trans. Automatic Control, AC-12:290.

McKelvey, T. (1993). System identification using over-parametrized state-space models. Technical report, Report LiTH-ISY1454, Department of Electrical Engineering, Linköping University, S-581 83 Linköping, Sweden.

Moore, B. C. (1981). Principal component analysis in linear systems: controllability, observability, and model reduction. IEEE Trans. on Automatic Control, 26(1):17-32.

Mullis, C. T. and Roberts, R. A. (1976). Synthesis of minimal roundoff noise fixed point digital filters. IEEE Trans. on Circuits and Systems, 23(9):551-562.

Söderström, T. and Stoica, P. (1989). System Identification. Prentice-Hall International, Hemel Hempstead, Hertfordshire.

Thiele, L. (1984). Design of sensitivity and round-off noise optimal state-space discrete systems. Circuit Theory and Applications, 12:39-46.

Thiele, L. (1986). On the sensitivity of linear state-space systems. IEEE Trans. on Circuits and Systems, 33:502-510.

van Overbeek, A. J. M. and Ljung, L. (1982). On-line structure selection for multivariable state space models. Automatica, 18(5):529543.

Van Overschee, P. and De Moor, B. (1992). Two subspace algorithms for the identification of combined deterministic-stochastic systems. In Proc. 31'st IEEE Conference on Decision and Control, Tuscon, Arizona, pages 511-516. 